\title{
Shifting college students' epistemological framing using hypothetical debate problems
}

\author{
Dehui $\mathrm{Hu}^{1,2}$ and N. Sanjay Rebello ${ }^{1}$ \\ ${ }^{1}$ Department of Physics, Kansas State University, Manhattan, Kansas 66506, USA \\ ${ }^{2}$ School of Physics and Astronomy, Rochester Institute of Technology, Rochester, New York 14623, USA
}

(Received 15 May 2013; published 27 May 2014)

\begin{abstract}
Developing expertise in physics problem solving requires the ability to use mathematics effectively in physical scenarios. Novices and experts often perceive the use of mathematics in physics differently. Students' perceptions and how they frame the use of mathematics in physics play an important role in their physics problem solving. In this study, we examined students' epistemological framing about using mathematics in physics in two types of problems: a conventional problem and a hypothetical debate problem. We found that when solving a conventional physics problem, students tended to frame problem solving in physics as rote equation chasing, i.e., plugging quantities into a memorized physics equation. In hypothetical debate problems, students were more likely to be involved in quantitative or qualitative sense making. We conclude that hypothetical debate problems might be used as an instructional tool for engaging students in sense making while using mathematics in physics. Thus, it might be potentially useful for developing more expertlike problem solving expertise.
\end{abstract}

DOI: $10.1103 /$ PhysRevSTPER.10.010117

PACS numbers: 01.40.-d

\section{INTRODUCTION}

Mathematics is regarded as the backbone of physics and science in general. Physics classes often involve the intensive use of mathematics, and it requires much more than the straightforward application of mathematical operations and algorithms. Physics problem solving requiring mathematics could be modeled as the following sequence of steps: mapping a physical situation onto a mathematical model, processing mathematical representations, interpreting mathematical results in physics, and evaluating whether the results adequately describe the physical system $[1,2]$. A significant portion of students in introductory level physics courses often struggle to apply mathematical skills in physics problem solving $[3,4]$. Research has shown that novice students often perceive the use of mathematics in physics very differently from expert physicists [1] and their expectations or perceptions can play powerful roles in their problem solving in physics [5,6]. Students' expectations about what they are supposed to do in physics problem solving could strongly affect or even control their selection of knowledge resources in a situation. In this study, we investigate students' physics problem solving from the perspective of epistemological framing, which describes an individual's perceptions about what knowledge tools or skills are appropriate to use in a learning situation [6,7]. The main goal of this study is to investigate how

Published by the American Physical Society under the terms of the Creative Commons Attribution 3.0 License. Further distribution of this work must maintain attribution to the author(s) and the published article's title, journal citation, and DOI. hypothetical debate problems (HDPs) [8,9] affect students' epistemological framing in physics problem solving.

Hypothetical debate problems require students to compare discrepant problem solving strategies presented by hypothetical problem solvers and discuss why they agree or disagree with each problem solving strategy. The hypothetical solution strategies provided in the debate problem are developed from common students' approaches to solving the problem. They are designed to help students develop their understanding by sharpening student awareness of their own and other students' ideas as well as attempting to explain discrepant strategies. We expect that when working on debate problems students are more likely to make sense of concepts rather than manipulating symbols and equations. Thus, we aim to answer the following research question: Does working on a hypothetical debate problem shift students' epistemological framing compared to working on a traditional problem?

In the following section, we describe our theoretical stances of epistemological resources and framing. We start with an overview of previous research on resources and framing and then we specifically talk about students' epistemological framing related to physics problem solving requiring the use of mathematics. In Sec. III we discuss the rationale of our instructional strategy-the development of hypothetical debate problems. The research design and data analysis is presented in Sec. IV. Then the research findings are presented in Sec. V. Finally in Sec. VI we present our conclusions and provide a discussion about the significance of this research on physics learning and instruction. 


\section{THEORETICAL BACKGROUND: EPISTEMOLOGICAL RESOURCES AND FRAMING}

The knowledge-in-pieces or resource-based $[5,10]$ perspective provides the theoretical stance for this research. We begin with a brief overview of the resources framework and then talk about how the epistemological framing fits within this framework. In particular, we will focus on the research about students' epistemological framing while using mathematics in physics problem solving.

\section{A. Resources framework}

In physics education research, there are two main approaches for modeling and characterizing student thinking: the unitary, misconception-based approach $[11,12]$ and the manifold or knowledge-in-pieces approach [10]. The central idea of the unitary framework is that students possess robust cognitive structures or misconceptions that need to be torn down in order to build the correct conception [11,12]. The manifold or knowledge-in-pieces framework is based on the assumption that one's conception is determined by activation of various resources depending on the context [13]. The knowledge-in-pieces approach has been proven useful for modeling student reasoning and learning in a growing body of evidence about the flexibility and variability in student reasoning $[5,14,15]$.

Consistent with the knowledge-in-pieces perspective, cognitive resources are fine-grained knowledge elements that one brings into a situation, and the activation of one resource often depends on context [13,14]. Typically, we think of resources as chunks of knowledge elements that can be applied productively in many situations. Resources might be unproductive for learning physics if used inappropriately in a situation. Resources are often associated with each other as a network, and the activation of one resource often leads to the activation of other resources or clusters of resources. When the same locally coherent set of resources becomes activated again and again, it will eventually be strongly connected and established to be activated as a unit. Learning physics largely entails the activation of existing resources, including conceptual [14,16], procedural [17], epistemological [18], and other types of resources. Epistemology involves the understanding of the nature of knowledge and how it is obtained [14]. Epistemological resources control how a student perceives the nature of learning in a situation and what conceptual resources are activated. For example, when learning physics many students appear to view physics knowledge as coming from authority. Like other resources, epistemological resources are context sensitive: they can activate or deactivate from moment to moment. An important part of learning physics involves the activation of appropriate epistemological resources in a certain context.

\section{B. Epistemological framing}

According to linguist Tannen [19], a frame describes an individual's expectations (i.e., What is it that's going on here?) about a situation and determines how one thinks and acts in a certain situation. Framing emphasizes the individual's interpretation of the situation as an ongoing process [20]. Under the resources perspective, framing is a cognitive process, which involves the activation of a locally coherent set of cognitive resources. Consider a situation in a lecture hall where a speech is going on: When entering a lecture hall, a student might recall their past experiences of listening to a lecture and think of "sitting quietly," "listening carefully," and "taking notes" as appropriate actions to make. In this example, a student's framing about this situation is based on the activation of a set of resources from past experiences while in the meantime framing determines how one reacts to the current situation. However, if the speaker stops lecturing and encourages the audience to discuss as a group instead, the student's original framing is challenged and not appropriate for this situation. The student probably feels nervous and uncomfortable when the situation challenges their expectations. Another possible situation might happen: a student activates a different set of resources related to their past experience of attending seminars or discussion groups. The student might reframe this situation as "making new friends" and "exchanging ideas with others." Hence, framing is a dynamic process, a part of an individual's cognition in response to an external environment, such as the speaker's tone, a question from the audience, or even a spontaneous idea jumping into one's mind. An individual will reframe their own activity upon receiving new information.

Framing has many components including social ("Who am I interacting with?"), affective ("How do I expect to feel about it?"), epistemological ("What do I expect to use to answer this question?"), and others [6,15]. Epistemological framing describes one's perceptions or judgments about what knowledge tools could be used in a learning situation. Students' expectations about what they are supposed to do play an important role in deciding what they should pay attention to and what knowledge resources they will use for creating new knowledge. In a physics class, a student might frame learning physics as "remembering the knowledge from an authority." The instructor might expect students to construct new knowledge by connecting physics concepts to their everyday experiences. Hence, students activate a different set of epistemological resources than the teacher does. Communication problems can arise when students and the teacher frame a situation differently during an interaction.

When solving physics problems, a student may frame a physics problem as an opportunity for making sense of concepts or an occasion for manipulating formulas and numbers [21]. Students' epistemological framing affects 
the ways in which they apply mathematics and interpret mathematics in physics. Tuminaro [4] observed student groups solving problems in an algebra-based physics course and identified three common frames in which students solving physics problems required the use of mathematics known as rote equation chasing, qualitative sense making, and quantitative sense making. The rote equation chasing frame is when students expect problem solving in physics to involve plugging quantities into a memorized physics equation. The qualitative sense-making frame is when students expect physics problem solving to include the application of physics principles or common sense, but they are devoid of formal mathematical procedures or equations. Finally, in the quantitative sensemaking frame, students expect that the solution requires formal mathematics integrated with physical sense making. The three common frames identified by Tuminaro [4] provide a basis for comparing student behavior in conventional and debate problems.

Bing and Redish [7] analyzed and classified students' framing about using math in physics by classifying the reasons or warrants students provide to support their points of view. The warrant analysis could be a useful tool for finding evidence of students' epistemological framing, which is typically implicit in their work. For instance, in "calculation" framing, the student relies on mathematical computations and the unspoken warrant might be that the student would accept a result by carefully following a set of computational steps. In "physical mapping" framing, students would make a claim or accept a result based on the evidence that mathematical calculations are consistent with a physical mechanism or their common sense about the physical scenario. The warrants from student work are associated with the epistemological resource activated at that moment: "a mathematical symbolic representation faithfully characterizes some feature of the physical or geometric system it is intended to represent" [7]. The researchers can get information about students' tacit epistemological resources activated in their mind or epistemological framing processes by identifying the warrants involved in their work.

In summary, we have introduced previous studies on resources and framing, as well as epistemological resources and framing, in particular. This study is concerned with students' epistemological framing about physics problem solving. The classification and warrant analysis about students' framing provides us with a thorough understanding of students' judgments regarding physics problem solving beyond the scope of conceptual learning. As instructors, we are interested in helping students take more productive approaches to learning and frame physics problem solving as sense making rather than rote use of formulas. Previous research has suggested instructional strategies to facilitate the shifts of student framing, such as manipulating the wording and representation of the problems, changing the classroom setting, and modifying the structure of the lecture [15]. In this study, we introduce a new type of problem - a hypothetical debate problemand examine students' framing in both a conventional problem and a hypothetical debate problem. We use the three common frames described in Tuminaro's [4] work and the tool of warrant analysis in the work of Bing and Redish [7].

\section{RATIONALE FOR HYPOTHETICAL DEBATE PROBLEMS}

The hypothetical debate problem is a new type of problem that differs from the conventional problems we encounter in physics. A conventional physics problem typically involves a physical scenario and a question at the end. To solve a conventional problem, students need to find an answer to the question based on given information in the problem scenario. When working on hypothetical debate problems, the goal is to find out the correct or incorrect strategies and figure out why or where the incorrect strategies are incorrect. A major difference between a conventional problem and a hypothetical debate problem is that a HDP typically requires students to compare several problem solving strategies or types of reasoning that are often contradictory instead of asking students to create a solution by themselves. Those problem solving strategies provided are often from common students' answers. The structure of a HDP creates an imaginary scenario of "debating with peers," through which students' own knowledge structures are challenged and reevaluated.

The development of a HDP is based on the pedagogical strategies of cognitive conflict and contrasting cases as well as empirical evidence from our earlier studies. Cognitive conflict is derived from a Piagetian view of learning in which the learners' active part in reorganizing their knowledge is central to learning [22]. Instructional approaches based on cognitive conflict involve promoting situations where the students' existing ideas about some phenomenon are made explicit and are then directly challenged in order to create a state of cognitive conflict [23].

When solving physics problems, students often tend to focus on the surface features of the problems and develop an overly superficial understanding of the concepts, limiting their ability to transfer to new situations [24]. Perceptual learning is the process of learning the skills of perception (i.e., noticing and differentiating), and it emphasizes the role of attention in people's learning process $[25,26]$. Based on the theory of perceptual learning, contrasting cases are important to guide people to notice and differentiate knowledge and experiences by affecting what one notices about subsequent events and how one interprets them [26,27]. Garner [26] provided an example to illustrate the role of contrasting cases in affecting noticing. First, he asked readers to look at a figure (i.e., 


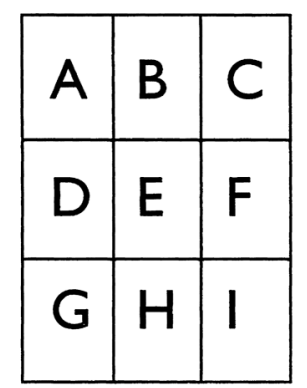

FIG. 1. Standard figure.

standard figure in Fig. 1), and then asked them to describe the figure. Most people described it as a grid with letters and some described it as a set of lines. Then the same figure was shown alongside another figure (Fig. 2). The new features such as size, shape, and symmetry became salient and relevant to the readers. Hence, by comparing and contrasting these two figures, they allowed readers to attend to distinguishing features and filter out irrelevant features [25]. Bransford and Schwartz [27] also pointed out that analyzing contrasting cases could help learners generate the differentiated knowledge structure that enabled them to understand a concept or a phenomenon deeply.

In summary, the instructional strategies of cognitive conflict and contrasting cases can serve two functions. First, by comparing and differentiating discrepant cases (reasoning or strategies), learners are cued to notice the deep structure that distinguishes the cases rather than the superficial features. Second, when confronting a different strategy, learners' own knowledge structures are challenged and reorganized to resolve the conflict. Compared to conventional problems, hypothetical debate problems can potentially shift students' attention away from pattern matching of existing examples.

Problem tasks similar to HDPs have been used effectively in previous studies to help students construct conceptual understanding $[28,8]$ as well as to investigate students' deep understanding of math and physics concepts [9]. In our study, the main purpose is to facilitate students to be engaged in sense making instead of the rote use of equations while applying mathematics in physics problems. To facilitate this change of frame, we present students with
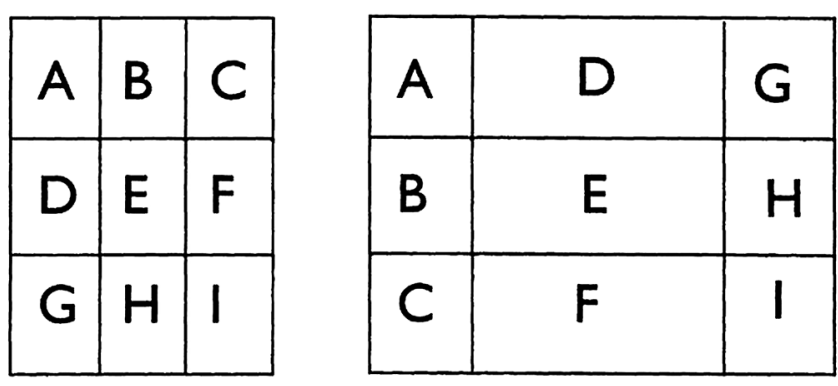

FIG. 2. Standard figure in the context of another figure. several problem solving strategies, some of which are based on commonly used albeit unproductive student strategies based on equation manipulations and others based on more sophisticated, productive strategies based on conceptual understanding of the mathematics and physics. We aim to steer students' attention away from strategies that focus on mathematical manipulations to those that focus on the deep structure of mathematics and physics concepts.

\section{METHODOLOGY}

\section{A. Data collection}

We conducted four pairs of 75-minute-long group interviews with 13 participants selected from a pool of 40 volunteers in a second-semester calculus-based physics course for engineers at a Midwestern university. The participants were selected based on convenience of scheduling. Each interview occurred within one week after students covered the related concepts in class. All participants had taken the prerequisite mathematics course (i.e., calculus of single variable). Students were organized in five groups of two or three students each. During the interviews, students discussed problems together on a whiteboard and the interviewer occasionally asked students to elaborate on their thinking. In the first interview of each interview pair, students worked together on three conventional physics problems requiring mathematical integration. An example is shown in Fig. 3. In the second interview, students worked on a problem similar to the first interview problem, but the problem was presented in a hypothetical debate format. The second interview of each pair occurred within one week after students completed the first interview. An example of the hypothetical debate problems is given in Fig. 4.

The hypothetical debate problem shows conflicting strategies employed by four hypothetical students (A-D). These strategies were created based on two resources: strategies documented in previous studies [29] and strategies proposed by participants in the first interview. Students were asked to compare those conflicting strategies and talk about why they agree or disagree with those ideas.

On average, students spent around 20 minutes on each problem. In this paper, we show analysis of student work on two problems based on the same problem context which was given in the first and second interview sessions. In the first interview, students solved a conventional problem (Fig. 3). We analyzed students' solutions for all groups given this problem to create four hypothetical strategies (A-D) for the debate problem in Fig. 4. There are two main reasons for selecting this problem for analysis. First, it was a relatively easy physical scenario and the problem is not based on rote memorization. Thus, less help was needed from the interviewer regarding the physics concepts so that we could neglect the effect of student-interviewer interaction on their framing. Second, this problem was not similar to problems that students had just encountered in 
A thin disk with radius $\mathrm{R}$ has a non-uniform surface charge density $\sigma r=\alpha r$ ( $\alpha$ is a constant). Find the total charge on this thin disk.

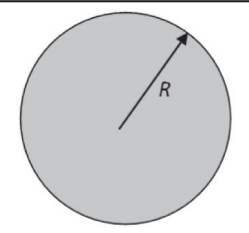

FIG 3. Example of a conventional problem.

their class, so it required them to construct their own solutions rather than directly apply formulas from memory.

\section{B. Data analysis}

We watched the video data to look for evidence of how students frame physics problem solving and identify the moments when there was a shift in students' framing. Students' framing can shift from moment to moment, and in this analysis it varies from several seconds to several minutes. Our data analysis was primarily based on the three kinds of frames identified by Tuminaro [4]. The correctness or incorrectness of student reasoning was not the focus of this study. We also recognize the work of Bing and Redish [7] and summarize the evidence that indicates students' framing about mathematics use in our problem solving activity in Table I.
In the rote equation chasing frame, students perceive physics problem solving as plugging quantities into a memorized physics equation. This frame shares some similarity with the "calculation" frame in the work of Bing and Redish, in which students rely on mathematical computations. Bing and Redish identified some common indicators of students' "calculation" frame, such as "correctly following algorithmic steps gives trustable result" and "focus on technical correctness." In our data analysis we identified some common student behaviors as an indication of a "rote equation chasing" frame, including "focus on algorithmic steps," "plug physical quantities into a memorized equation," and "map physical quantities to the solution of a memorized example." Bing and Redish also identified the "physical mapping" frame, meaning that students would make a claim or accept a result based on the evidence that mathematical calculations are consistent

A thin disk with radius $R$ has a non-uniform surface charge density $\sigma(r)=\alpha r$ ( $\alpha$ is a constant). Find the total charge on this thin disk. Four students were discussing how to solve this problem and they provided four different strategies. Now discuss in a group why you think each strategy (or part of the strategy) is correct/incorrect.

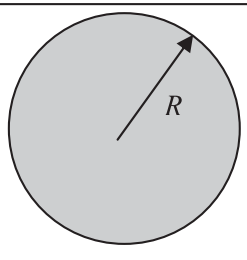

Student A: The surface charge density is charge per unit area, thus, the total charge is the surface charge density times area, that is $Q=\sigma(r) \cdot A . \sigma$ changes with $\mathrm{r}$, so an integral is needed here.

The final equation should be $\int_{0}^{R} \sigma(r)\left(\pi R^{2}\right) d r=\pi R^{2} \int_{0}^{R} \sigma(r) d r$.

Student B: Because we need to add the areas of the rings, $A=\pi r^{2}$ and it should be inside the integral. Thus, the total charge is $\int_{0}^{R} \sigma(r)\left(\pi r^{2}\right) d r$.

Student C: As the surface charge density changes with $r$, we need an integral. First, we need to chop the whole disk into infinite numbers of extremely thin rings with thickness $d r$. For an arbitrary thin ring located at a distance $r$ from the center, it has an area of $d A=2 \pi r d r$, and $\sigma(r) d A$ gives the small amount of charge carried by this ring. The total charge is the summation of all the charges carried by each ring, that is $\int_{0}^{R} \sigma(r) d A=\int_{0}^{R} \sigma(r) 2 \pi r d r$.

$\underline{\text { Student D: }}$ The surface charge density is changing, the integral $\int_{0}^{R} \sigma(r) d r$ means averaging up the charge density along the radius from 0 to $R$. Then we just apply it into the equation $Q=\sigma A$ for a constant charge density. Thus, the total charge is $\left(\int_{0}^{R} \sigma(r) d r\right) *\left(\pi R^{2}\right)$.

FIG. 4. Example of a hypothetical debate problem. 
with a physical mechanism or their common sense about the physical scenario. This is similar to the "quantitative sense-making" frame in Tuminaro's work, in which students expect that the solution requires formal mathematics integrated with physical sense making. According to the main features of a quantitative sense-making frame, we identified some common student behaviors, including "map a physical situation to a mathematical equation" and "interpret mathematical symbols or equations in a physical scenario." In the qualitative sense-making frame students expect physics problem solving to include the application of physics principles or common sense without the use of formal mathematical equations. Hence students' behaviors include "use qualitative analysis" and "draw diagrams or use words to explain physical mechanism."

As students were solving the problem, the interviewer interacted with students on very few occasions and only to clarify the questions for the students. Since the interviewer did not significantly interact with the students, our analysis does not include the time when they were interacting with the interviewer. We also excluded data when it was not possible to identify students' framing, including students reading the problem statement, asking clarification questions, and talking about something irrelevant. This fraction of time is very small (about 6\%) while students were solving the conventional problem, compared with $25 \%$ for hypothetical debate problem, when students spend more time on reading the problem statement and strategies provided. Our unit of data analysis was a group. During the data analysis, we first identified the transitions of student framing and broke down the transcripts based on the transition moment. The timespan on each framing varies from 30 seconds to about 2-3 minutes. The transitions were identified based on the indicators of each type of framing given in Table I. The time tolerance for considering the same codes is around 5 seconds. We randomly selected two episodes of about 10 minutes each to check the coding reliability and the interrater reliability is about $85 \%$-the percentage of time in which the codes from two separate people were the same. The time tolerance for considering the same codes was around 5 seconds. We aggregated the amount of time that students spent on each frame and then compared the percentage of time for each frame on both the conventional problem in interview one and the hypothetical debate problem in interview two.

\section{RESULTS AND DISCUSSION}

We found that students' framing about solving a physics problem is different on the conventional physics problem; overall, students spent significantly less time on rote equation chasing when working on the hypothetical debate problem. Among the five groups there were two groups of students who spent less than $30 \%$ of their time on the rote equation chasing frame while solving the conventional problem. During this time, students were mainly focused on doing calculations and reorganizing equations, which are also a necessary part of solving physics problems requiring mathematics. Their entire problem solving activity involved frequent use of diagrams, graphs, gestures, and verbal explanations. In other words, their frames about physics problem solving are very productive and similar to those of experts. In one of the other groups, one student figured out how to solve the problem in about 2 minutes and explained his strategy to the rest of the group who seemed to agree with him. Thus, the time for student problem solving activity was too short for us to identify the shift in their framing.

In this study we were interested in students who were involved in a less productive (i.e., rote equation chasing) frame when solving a conventional problem. Our hypothesis was that the use of a hypothetical debate problem might help these students, who tended to favor rote equation chasing, to shift their frame to a more productive sense-making frame. To demonstrate how the framing of these students shifted as they moved from a conventional

TABLE I: Three frames and their indicators.

\begin{tabular}{|c|c|}
\hline Frame & Evidence of frame \\
\hline $\begin{array}{l}\text { Rote equation } \\
\text { chasing }\end{array}$ & $\begin{array}{l}\text { Focus on algorithmic steps; plug physical quantities into a memorized equation; } \\
\text { map physical quantities to the solution of a memorized example } \\
\text { Example: Using a memorized equation } \sigma=Q / A \text { and plugged in physical quantities } \\
\text { without considering whether the equation is applicable or not }\end{array}$ \\
\hline $\begin{array}{l}\text { Quantitative } \\
\text { sense making }\end{array}$ & $\begin{array}{l}\text { Map a physical situation to a mathematical equation; interpret mathematical symbols } \\
\text { or equations in a physical scenario; sometimes aided by a diagram or gesture } \\
\text { Example: Trying to interpret which area } \pi R^{2} \text { represents }\end{array}$ \\
\hline $\begin{array}{l}\text { Qualitative } \\
\text { sense making }\end{array}$ & $\begin{array}{l}\text { Use qualitative analysis without formal mathematics; often draw diagrams or use } \\
\text { words to explain physical mechanism } \\
\text { Example: Talking about adding small amount of charges to get the total charge } \\
\text { without using formal mathematical equations }\end{array}$ \\
\hline
\end{tabular}


problem to a hypothetical debate problem, we present the results for the two groups of students who were primarily involved in a rote equation chasing frame on the conventional physics problem and provide a detailed discussion about their framing for both types of problems to provide evidence for frame shifting. We aggregated the amount of time that both groups of students were involved in each frame and compared the percentage of time for each type of framing as shown in Fig. 5.

We conducted a statistical analysis of the data presented in Fig. 5 using the nonparametric Fisher's exact test. We used this test because of the small sample sizes in our study. Comparing the percentage of time students spent using each strategy, we find there is a statistically significant difference between those who solved the conventional problem and those who solved the debate problem: $\chi^{2}(2)=41.22, p<0.001, V=0.45$. The $p$ value indicates that the probability that the two problem types are equivalent in terms of the percentage of time spent in different frames is less than 0.001. The effect size of Cramer's $V=0.45$ indicates a moderate effect. Further, we find that students solving the conventional problem spent significantly more time rote equation chasing and significantly less time using quantitative and quantitative sense making than those who solved the debate problem.

When working on the conventional problem, students were engaged in rote equation chasing about $66 \%$ of the time during their problem solving activity, compared with only $20 \%$ or less time on qualitative or quantitative sense making. We found that students often tried different mathematical equations based on their memorization and plugged physical quantities into the equations to get an answer without considering whether the equations were appropriate in this context. We selected an episode from one group of students to illustrate what students typically do as they solve the conventional physics

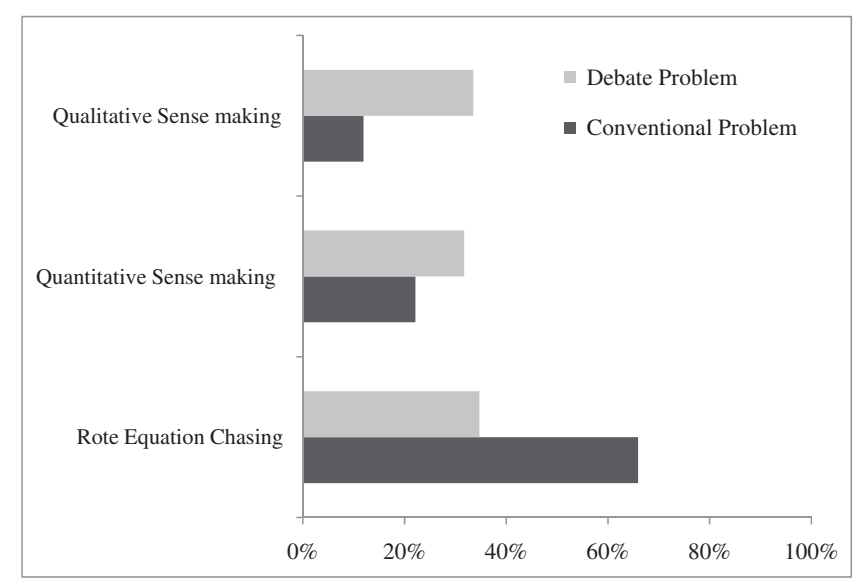

FIG. 5. Percentage of time students were involved in each frame. problem. The following conversation occurred at the beginning of this problem solving activity. In this episode, students frequently used particular words, such as "equals," "multiply," and "take the integral," indicating that they primarily focused on manipulating mathematical symbols and equations. We emphasize several key words and provide a detailed description of how the use of those words is related to students' framing in our analysis below.

Alan: Can't we just say if $\alpha r$ equals our charge density and our charge density equals $Q$ over $A$ ? Can we say $\alpha r$ equals $Q / A$ ? Just multiply this (charge density $\alpha r$ ) by $A$, and we get $Q$ - our charge.

Zad: Well, write that out.

Then Alan started to write down equations as shown in Fig. 6.

Alan: Because this $(\alpha r)$ equals our charge density given by that, you just multiply A and then you get a charge.

Zad: Okay. And then take the integral of that, over the area, from 0 to $2 \pi$.

Alan: So the area would be, area of our circle is $2 \pi \ldots$ Phil: $2 \pi r^{2}$.

Alan then replaced $A$ by $2 \pi \mathrm{r}^{2}$ in the second equation of Fig. 6.

Alan: Right?

Phil: Uh huh.

Alan: So we want to integrate that for $r$ ? From 0 to $R$ ?

Zad: We have several equations where we have charge density, substitute that in, um, charge density, charge over area. We have defined the full circle of the radius, the charge, the derivative of the charge, so we find it out at a certain point, times the area, so we integrate it, uh, over an area, so we integrate it.

In this episode all three students were focused on how to find the expression for total charge through manipulating the physical quantities and mathematical equations. At the beginning, Alan recalled an equation (i.e., charge density $=Q / A$ ), which related the quantities charge density and total charge. When realizing that the charge density is charge over area, Alan set the charge density function $[\sigma(r)=\alpha r]$ "equals" $Q / A$. Then he rearranged this new equation to find the total charge $Q$ as shown in Fig. 6.

As soon as Alan recalled the mathematical equation charge density $=Q / A$, he related this equation to the given



FIG. 6. Equations set up by students in the conventional problem. 
information charge density function $\sigma(r)=\alpha r$, and set up a new equation $\sigma(r)=Q / A$. At the beginning of this episode, the word "equal" appeared three times. It seemed like Alan's strategy was simply based on the equal sign: both $Q / A$ and $\alpha r$ equal charge density, thus, those two quantities should equal. There was no evidence that Alan considered whether or not this equation charge density $=Q / A$ was appropriate to use in this context, or what the charge density represented. Once he obtained an equation $[\sigma(r)=Q / A]$, he multiplied $\sigma(r)$ by $A$ to find the total charge. While explaining his work, Alan frequently used the words equal and multiply, which indicated that Alan treated this physics problem as manipulating mathematical symbols and equations. Later on, Zad proposed the use of integration and eventually set up an integral $\int_{0}^{2 \pi} \sigma / 2 \pi r^{2}$ although he was not sure whether it was right or wrong. He started out with "substitute in" the given charge density equation to the integral template and then integrated "over an area." He did not talk about what physical quantities to integrate or which area to integrate as represented by the equation. Overall, this group of students focused primarily on the mathematical operations and did not pay attention to the physical meaning of mathematical notations used in an equation. They seldom talked about how to map the physical situation onto the mathematical equations or the meaning of mathematical equations. Hence, students were mainly involved in a rote equation chasing frame in this episode.

Comparing students' framing on conventional and debate problems in Fig. 5 clearly shows that while working on a debate problem, the percentage of time that students spent on rote equation chasing decreased from $66 \%$ to $35 \%$ and their framing shifted to either qualitative or quantitative sense making. In Fig. 5, we also observe a tendency that students' framing shifted from rote equation chasing to qualitative sense making. The percentage of qualitative sense making is much higher while solving a hypothetical debate problem (36\% vs $12 \%$ ). When working on a debate problem, they often focused on comparing one strategy with another, discussing how those strategies were different, and which strategy "makes more sense." We also selected an episode from the same group of students as they worked on the hypothetical debate problem. The words that the students chose were quite different, compared with the first episode. They talked more about the physical picture and the physical meanings of symbols instead of mathematical computation. Again, we have emphasized particular words that could reflect students' framing of the activity.

Zad: The difference between [students] A and B is A is taking area times the charge of each...

Phil: Each ring?

Zad: I think I was wrong.

Alan: So we need to do some dA, not just A.

Zad: No, you don't have to do the derivative of A, because you can do that as a function. Yeah, cause you can do that as a function of this.

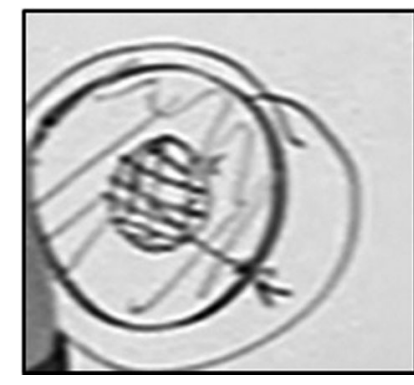

FIG. 7. Picture drawn by students in the hypothetical debate problem.

Because we... I... drew this circle (Fig. 7), this is $\mathrm{R}$, when you find it, when you're finding this little area, (shaded the area of the small circle as shown in Fig. 7).

Alan: A small section would be dA (pointed to the small circle in Fig. 7).

Zad: The radius is $R$, you are not times the radius of the whole (drew the big circle in Fig. 7), you times the radius of that area (shaded the area of the small circle again). Because that wouldn't make sense to find the charge of this (pointed to the small circle) and times the area of this all (shaded the area of the ring between two circles).

Alan: So you are looking at some r, some small radius, times some small area $d A$.

Zad: Yeah.

In the above episode, Zad started with comparing strategies $\mathrm{A}$ and $\mathrm{B}$ and attempted to understand the difference between the mathematical equations used in strategies $\mathrm{A}$ and $\mathrm{B}$. When comparing different strategies, the structure of equations became more evident to students. As an example, in this episode, students started to pay attention to the use of a specific symbol and tried to understand how expression $\pi R^{2}$ in strategy A was different from $\pi r^{2}$ in strategy B. To help them understand what each mathematical expression represented, Zad then drew a picture (Fig. 7) of the circle of charge to help other members of his group visualize the area. When working on hypothetical debate problems, students tended to draw diagrams or use verbal explanations instead of drawing from their memorization of mathematical equations or solutions from past experiences. With the help of this diagram, they were able to draw the "radius of the whole" and the "radius of that area" on the diagram to differentiate symbols $R$ and $r$; they also pointed to "a small section," "little area," and "area of this all" on the diagram while interpreting the meaning of symbol $d A$. As students talked about "charge of this (pointed to the small circle) and times the area of this all (shaded the area of the ring between two circles)," they appeared to focus on the physical scenario rather than less concrete mathematical symbols. As students got to the deep structure of the equations, they started to realize that they should use "some small area $d A$," and not the "area of this all." 
In the previous episode, when asked to invent their strategies to solving this problem, students only applied the simple rule that "total charge is equal to charge density multiplied by area," without paying attention to which area the mathematical equation represents. When Zad said "the radius is $R$, you are not times the radius of the whole, you times the radius of that area," he seemed to be debating with a fictitious student, explaining what the equation represented in order to convince the peer why his or her solution was wrong. Their explanations were accompanied by embodied gestures such as drawing a circle or shading an area. While working on hypothetical debate problems, this group of students mostly used qualitative approaches and tools such as drawing a diagram, explaining the physical meaning verbally, and body gestures. We identified that students were primarily involved in a qualitative sense-making frame.

Overall, we found that both groups spent the majority of time on a rote equation chasing frame while working on the conventional problem. They all seemed to expect solving physics problems as a direct application of formulas or "doing math." However, when the problem was presented in a different format, instead of finding an "answer" they were asked to "debate with fictitious peers" so their framing shifted from rote equation chasing to sense making on the hypothetical debate problem.

In discussing students in qualitative or quantitative sense making, we do not claim that one is better than the other. Problem solving mainly involves the correct use of mathematics (i.e., the mathematical concept of integral) in a physical situation, and using mathematical equations is a necessary part of this problem solving activity. When working on the hypothetical debate problem, students compared the difference between the mathematical equations, and the structure of mathematical equations often became more salient to students. Hypothetical debate problems seemed to make students move their attention away from the superficial features of equations (e.g., whether or not a mathematical equation is simplified) and motivated the students to explore the underlying meaning of the mathematical equations. Students also tried to make use of multiple representations to explain the meanings of mathematical equations in order to convince the fictitious peers. We found that students spent about $36 \%$ of the total time on qualitative sense making. Given that this problem cannot be solved using a purely qualitative approach, with no formal mathematics, we should not expect that percentage to be very high.

\section{CONCLUSIONS AND IMPLICATIONS}

In this paper we analyzed students' physics problem solving from the point of view of framing. We extended Tuminaro's [4] work on students' framing in algebra-based physics and applied his classification in calculus-based physics. Students' framings were investigated in two different problem types: the conventional free-response problem and the hypothetical debate problem. Though hypothetical debate problems have been used extensively in physics and science education in general, very few studies have carefully looked at the effectiveness of this new type of problem as an instructional tool. Our data show that some students in our case study tend to frame their activity as rote equation chasing when solving the conventional problem. However, when working on the hypothetical debate problem, they were more likely to be in the quantitative or qualitative sense-making frame. Thus, our study shows that students appeared to frame the HDP task as one in which they were expected to engage in sense making rather than rote equation chasing. As students work on the debate problems, they seem to focus more on the deep structure of mathematical equations and trying to understand what they mean in a context rather than executing a mathematical procedure.

This work provides evidence for using hypothetical debate problems as an instructional tool for engaging students in mathematical sense making in physics from a framing perspective. We do not claim that the way students frame the use of math in physics is necessarily related to the correctness of their claims or responses. However, strategies that help engaging students in sense making could be potentially useful for developing expertlike problem solving strategies.

In order to build students' mathematical fluency in physics, instructors should be more aware of students' framings in a situation. This will help us determine whether a student's difficulty is due to a lack of particular knowledge or is a framing issue. From analyzing students' framing about physics problem solving, we can get information about their activated epistemological resources. For example, when a student frames solving physics problem as rote equation chasing, he or she might perceive using mathematics in physics as "doing mathematics." Previous studies (see Refs. [29,30]) have reported students' inability in using their mathematical skills in physics, and for a long time their difficulties were attributed to a lack of conceptual understanding of either mathematics or physics concepts. Later, research in physics education [31,32] pointed out that students often have the required mathematical skills; however, they were unable to apply these skills appropriately in a physics context. According to the resources [5] perspective, there are two possible explanations for students' deficiencies in physics problem solving requiring mathematics: students used resources unproductively in a context or students framed a situation in a problematic way, which in turn activated unproductive knowledge resources or skills. Recent research [4,7] has started to pay more attention to students' epistemological framing - expectations or judgments about how mathematics is used in physics. When an instructor is unable to ascertain students' framing of a situation, it can create a 
barrier in providing students the appropriate scaffolding to facilitate their learning.

The research reported in this paper shows that students seem to be more involved in qualitative or quantitative sense making when debating with fictitious peers. Thus, HDPs might be a useful instructional tool to engage students in sense making. In this particular study, we have used HDPs in the context of problems that require the use of mathematics in physics, and our results demonstrate that for the problems used in this study, the participants were more likely to engage in sense making while solving a HDF than a conventional problem. A follow-up study could be conducted to investigate if working through HDPs would improve students' understanding of mathematics and physics concepts, as well as the effect of HDPs on students' framing on conventional physics problem solving.

\section{ACKNOWLEDGMENTS}

This work is supported in part by the National Science Foundation Grant No. 0816207.
[1] E. F. Redish, in Proceedings of the Conference on World View on Physics Education in 2005: Focusing on Changing, Delhi, India, 2005 (unpublished).

[2] E. F. Redish and A. Gupta, in Proceedings of the GIREP-EPEC and PHEC 2009 International Conference, Leicester, England, (University of Leichester, UK, 2009) pp. 244-260.

[3] F. R. Yeatts and J.R. Hundhausen, Calculus and physics: Challenges at the interface, Am. J. Phys. 60, 716 (1992).

[4] J. Tuminaro, Ph.D. thesis, University of Maryland, 2004.

[5] D. Hammer, More than misconceptions: Multiple perspectives on student knowledge and reasoning, and an appropriate role for education research, Am. J. Phys. 64, 1316 (1996).

[6] E. F. Redish, in Proceedings of the International School of Physics "Enrico Fermi," Course CLVI (unpublished).

[7] T. J. Bing and E. F. Redish, Analyzing problem solving using math in physics: Epistemological framing, Phys. Rev. ST Phys. Educ. Res. 5, 020108 (2009).

[8] D. Nguyen, E. Gire, and N.S. Rebello, Facilitating strategies for solving work-energy problems in graphical and equational representations, AIP Conference Proceedings Vol. 1289 (2010).

[9] J. Von Korff and N. S. Rebello, Teaching integration with layers and representations: A case study, Phys. Rev. ST Phys. Educ. Res. 8, 010125 (2012).

[10] A. A. diSessa and B. L. Sherin, What changes in conceptual change?, Int. J. Sci. Educ. 20, 1155 (1998).

[11] M. T. H. Chi, Cognitive Models of Science, edited by R. Gierce, Minnesota Studies in the Philosophy of Science Vol. 15 (University of Minnnesota Press, Minneapolis, 1992), pp. 129-160.

[12] J. Clement, in Mental Models, edited by D. Gentner and A. Stevens (Lawrence Erlbaum, Hillsdale, NJ, 1983), pp. 325-339.

[13] A. A. diSessa, in Mental Models, edited by D. Gentner and A. Stevens (Lawrence Erlbaum, Hillsdale, NJ, 1983), pp. 15-34.

[14] D. Hammer, Student resources for learning introductory physics, Am. J. Phys. 68, S52 (2000).
[15] D. Hammer, A. Elby, R. E. Scherr, and E. F. Redish, in Transfer of Learning: From a Modern Multidisciplinary Perspective, edited by J.P. Mestre (Information Age Publishing, Greenwich, CT, 2005).

[16] M. C. Wittmann, Using resource graphs to represent conceptual change, Phys. Rev. ST Phys. Educ. Res. 2, 020105 (2006).

[17] K. Black and M. C. Wittmann, in Proceedings of the Physics Education Research Conference, Ann Arbor, MI, 2009 (unpublished).

[18] D. Hammer, and A. Elby, in Personal Epistemology: The Psychology of Beliefs about Knowledge and Knowing, edited by B. K. Hofer and P. R. Pintrich (Lawrence Erlbaum, Hillsdale, NJ, 2002), pp. 169-190.

[19] D. Tannen, in Framing in Discourse, edited by D. Tannen (Oxford University Press, New York, 1993), pp. 14-56.

[20] G. MacLachlan and I. Reid, Framing and Interpretation (Melbourne University Press, Melbourne, 1994).

[21] A. Elby and D. Hammer, Personal Epistemology in the Classroom: Theory, Research, and Implications for Practice (Cambridge University Press, Cambridge, 2010), pp. 409-434.

[22] J. Piaget, The Origins of Intelligence in Children (International Universities Press, New York, 1952), pp. 1-20.

[23] P. Scott and H. Asoko, Theoretical Issues and Empirical Studies: A Review of Strategies (1992), pp. 310-329.

[24] J. Larkin, J. McDermott, D. P. Simon and H. A. Simon, Expert and novice performance in solving physics problems, Science 208, 1335 (1980).

[25] E. J. Gibson, Principles of Perceptual Learning and Development (Appleton-Century-Crofts, Inc., New York, 1969).

[26] W. R. Garner, The Processing of Information and Structure (Lawrence Erlbaum Associates Potomac, MD, 1974).

[27] J. D. Bransford and D. L. Schwartz, Rev. Res. Educ. 24, 61 (1999).

[28] L. C. McDermott and P. S. Shaffer, Tutorials in Introductory Physics (Prentice-Hall, Upper Saddle River, NJ, 2002).

[29] V. Sealey, in Proceedings of the Twenty Eighth Annual Meeting of the North American Chapter of the International Group for the Psychology of Mathematics Education, Merida, Mexico, 2006 (unpublished). 
[30] L. C. McDermott, M. L. Rosenquist, and E. H. Van Zee, Student difficulties in connecting graphs and physics: Examples from kinematics, Am. J. Phys. 55, 503 (1987).

[31] L. Cui, N. S. Rebello, P. R. Fletcher, and A. G. Bennett, in Proceedings of the Annual Meeting of the National
Association for Research in Science Teaching, San Francisco, CA, 2006 (unpublished).

[32] D. Nguyen and N. S. Rebello, Students' difficulties with integration in electricity, Phys. Rev. ST Phys. Educ. Res. 7, 010113 (2011). 